Journal of Accident and Emergency Medicine 1995 12, 119-122
Correspondence:

\section{E. H. Kua,}

Associate Professor, Department of Psychological Medicine, National University Hospital, Lower Kent Ridge Road, Singapore 0511, Republic of Singapore

\title{
Psychiatric referrals from an accident and emergency department in Singapore
}

\author{
A.W.K. ANG, S.M. KO \& E.H.KUA
}

Department of Psychological Medicine, National University Hospital, Lower Kent Ridge Road, Singapore

\section{SUMMARY}

A total of 500 consecutive psychiatric referrals from the Accident and Emergency (A\&D) Department of the National University Hospital of Singapore were studied with regard to their demographic characteristics, diagnoses, presenting problems and management. There were 314 females (62.8\%) and 186 males $(37.2 \%)$, and the mean age of subjects was 35.5 years. The three main diagnoses were anxiety disorders (25.6\%), depression (19.4\%) and schizophrenia (17.6\%). About $41 \%$ were admitted, of whom two-fifths were initially admitted to the medical ward because of drug overdose or alcohol intoxication. A further $34 \%$ were treated as out-patients, and $21 \%$ were discharged.

Key words: emergency, management, psychiatry

\section{INTRODUCTION}

The Accident \& Emergency (A\&E) Department provides an important primary care function in urbanized cities, and the psychiatric service of the department has become a major point of entry into the mental health system. Studies of psychiatric morbidity levels at the A\&E department provide important information which could be useful in the planning and allocation of resources for efficient management of psychiatric disorders in a community.

While there have been several studies in the West, ${ }^{1-4}$ there have been no published reports from the Far East. In view of the significant socioeconomic changes in the Far East in the last decade, research on the utilization of the health services is clearly indicated.

The aim of this study is to provide data on A\&E psychiatric attenders, and to make comparisons with studies from the USA and the UK

\section{METHODS}

Singapore, an island-city, is a developing nation in South East Asia. The multi-racial population of 3 million comprises Chinese (77\%), Malays (14\%), Indians $(7 \%)$ and others $(2 \%)$. The National University Hospital (NUH) is the first general hospital in Singapore to provide a $24 \mathrm{~h}$ psychiatric consultation at the A\&E department. The A\&E staff consists of two consultants, one registrar and 14 medical officers. On each shift there are three medical officers, while the consultants and registrar are usually on the day shifts. There is also a psychiatric resident doctor on call on a daily basis, who may be called upon when a psychiatric referral is required for any patient at the A\&E department. Moreover, a psychiatrist, either a senior registrar or a consultant, is available for further consultation at any time. The Hospital serves a catchment area that includes people from all socio-economic classes and ethnic groups. In 1992, there were 78212 A\&E attendances, of which 860 (1.1\%) were psychiatric attendances. The diagnoses, based on the International Classification of Diseases, Ninth Revision (World Health Organisation, 1977), were made by the A\&E doctor or the psychiatric resident. The first 500 consecutive A\&E attenders with psychiatric diagnoses (ICD-9 codes 290 to 311) or diagnosis of poisoning by drugs (ICD-9 codes 960 to 977) were studied. Information on demographic characteristics, diagnoses, presenting problems and management were obtained from the case notes. The data were analysed using the SPSS software program on an IBM mainframe computer.

\section{RESULTS}

\section{Demography}

Among the attenders there were 314 females $(62.8 \%)$ and 186 males $(37.2 \%)$. The mean ages of 
the female and male attenders were 36.3 years (S.D. 12.6 years) and 34.2 years (S.D. 12.9 years), respectively ( $P=0.07$ using $T$-test). The frequency distribution of the sample is shown in Fig. 1. The majority of the patients were aged between 20 and 49 years. The ethnic composition of the sample was as follows: $82.7 \%$ Chinese; $4.0 \%$ Malay; $11.5 \%$ Indian, $1.8 \%$ other. Malays were under-represented compared to the other ethnic groups in the population.

\section{Diagnoses and symptoms}

The three commonest diagnoses were anxiety disorders $(25.6 \%)$, depression $(19.4 \%)$ and schizophrenia (17.6\%). Approximately half of the sample $(n=240)$ were suffering from non-psychotic conditions (NPC), i.e. depressive and anxiety disorders, and a quarter of the sample $(n=139)$ had a psychotic illness (PSY), i.e. schizophrenia, paranoid state or mania. Approximately $16 \%$ presented as drug overdose, and $5.6 \%$ for alcoholrelated problems.

The psychiatric diagnoses for the sample are illustrated in Fig. 2. The three commonest diagnoses in female and male attenders were similar, i.e. anxiety, depression and schizophrenia. Males were over-represented for alcohol-related problems and females for drug overdosage.
There were differences in the presenting complaints of patients in the NPC and the PSY groups. In the NPC group, the commonest complaints were somatic complaints $(47 \%)$, depressed mood $(33 \%)$, undue worries $(28 \%)$, sleep disturbances $(27 \%)$ and suicidal ideas or attempts (13\%). In the PSY group, the commonest problems were sleep disturbances $(37 \%)$, abnormal behaviour (32\%) (e.g. talking or laughing to oneself), hallucinations (22\%), delusional beliefs $(22 \%)$ and agitation (19\%). About $1 \%$ of the nonpsychotic patients and $10 \%$ of psychotic patients displayed aggressive behaviour.

\section{Management}

About one-fifth of the patients were discharged without follow-up, and one-third were referred for further out-patient follow-up. About two-fifths of the patients were admitted for treatment and, of this group, two-thirds were admitted to the psychiatric ward, mainly for treatment of depression or a psychotic disorder. Up to $70 \%$ of those admitted to the non-psychiatric wards had either drug-overdose or alcohol-related problems. About $3 \%$ of the patients who displayed violent behaviour were referred to the state mental hospital. Members of the NPC group of patients were more likely to be discharged without further

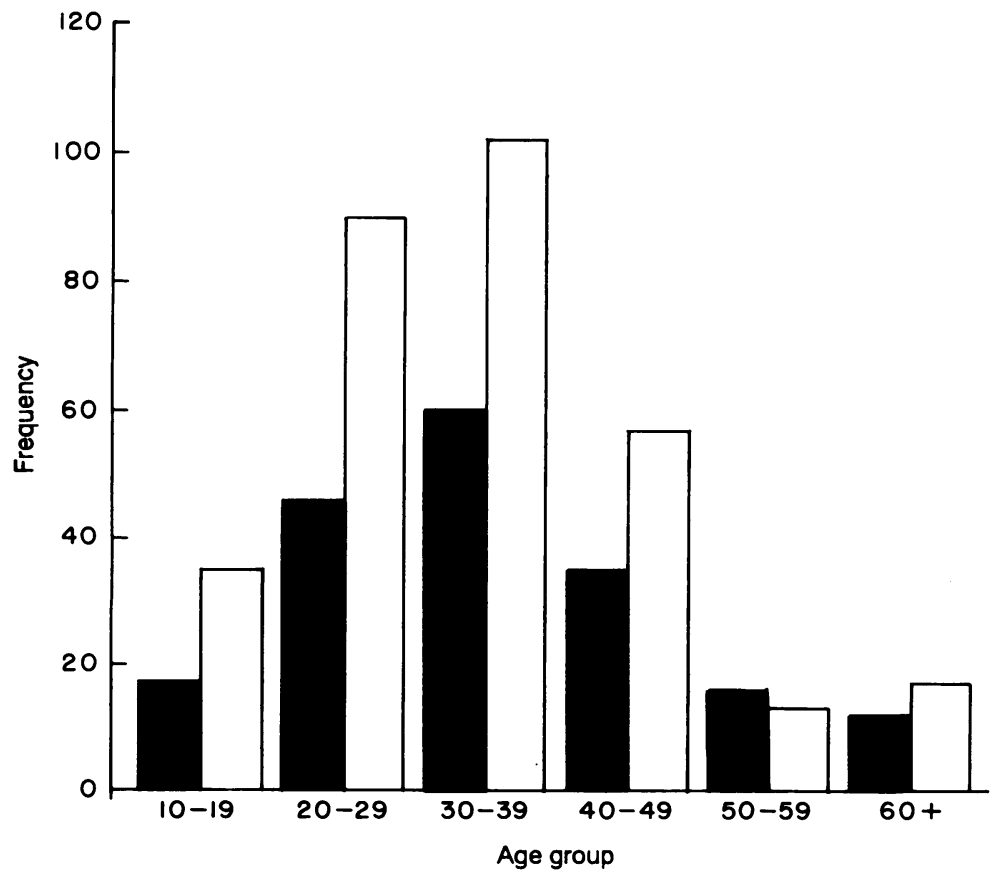

Fig. 1. Frequency distribution of the sample: age group by sex. 
Psychiatric

referrals from an $A$

\& E department

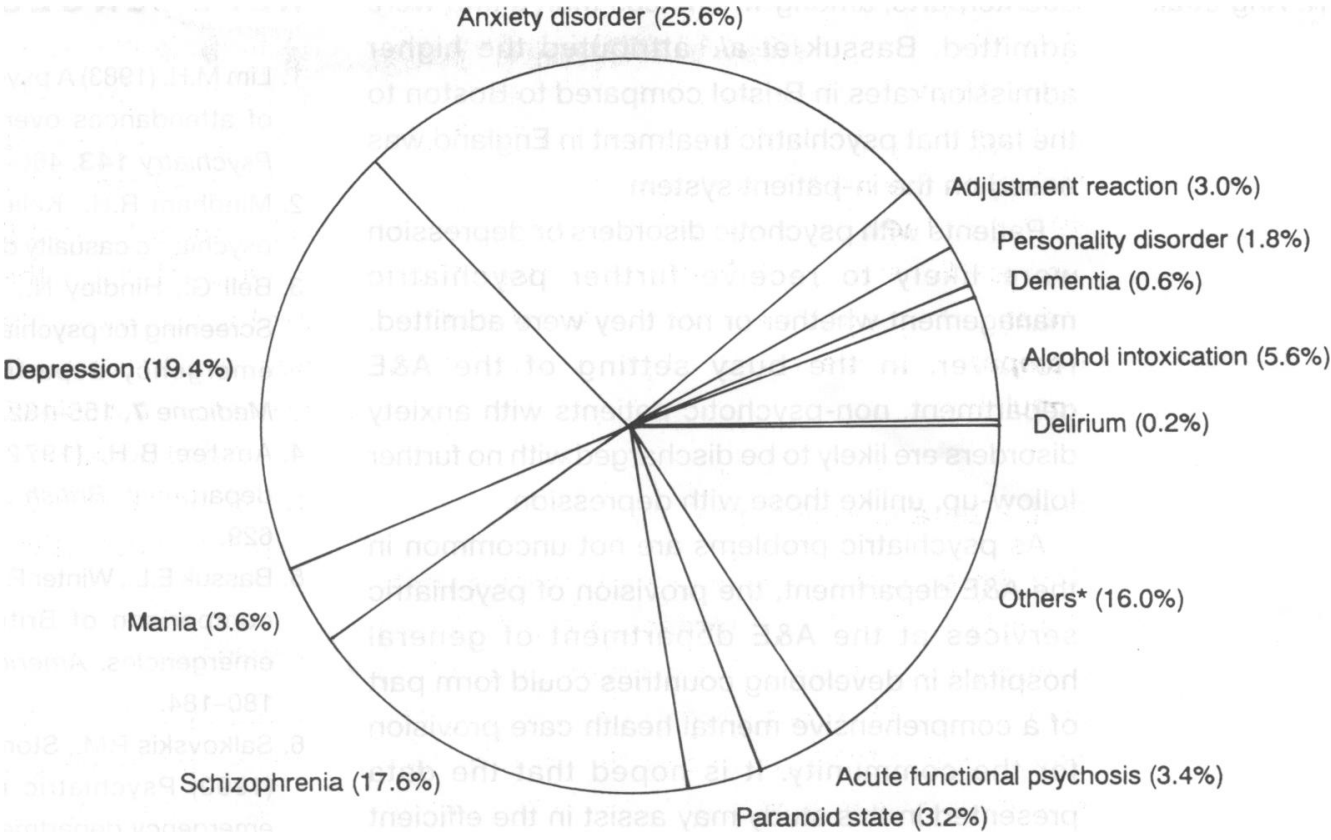

Fig. 2. Psychiatric diagnoses for the sample. follow-up than members of the PSY group (Chisquare $P=37.38 ; P<0.001$ ).

\section{DISCUSSION}

The sample of 500 consecutive A\&E psychiatric referrals from a general hospital (situated near to various public and private housing estates) may be considered a fairly representative sample of the psychiatric morbidity in the population that sought treatment in A\&E departments. This is in contrast to studies involving psychiatric attenders at private psychiatric clinics or the state mental hospital. The former would be biased towards patients from higher socio-economic classes, while the latter would over-sample patients from the lower social classes, or those exhibiting violent behaviour.

It is interesting to note that the Malay ethnic group, which represents $14 \%$ of the general population, formed only $4.0 \%$ of the sample. This suggests that, compared with the other ethnic groups, Malay patients with psychiatric disorders are less likely to seek treatment at the A\&E department. Spiritual healers (called 'bomohs') are often consulted for both physical and psychological symptoms in this group. During crisis, their strong support networks involving near and distant relatives are often activated, thus averting the need for A\&E attendance.

Comparing similar studies in other general 121 departments in Singapore is a third lower than that in Bristol and Boston. ${ }^{5}$ The fact that only $1.1 \%$ of total A\&E attenders received psychiatric diagnoses in our study does not necessarily imply a low rate of psychiatric morbidity in the A\&E department in Singapore. Using the General Health Questionnaire (GHQ) as a screening instrument for psychiatric morbidity in the A\&E department of a general hospital in London, Bell et al. ${ }^{3}$ found that $27.5 \%$ of A\&E attenders exhibited mild to moderate degrees of psychiatric morbidity, predominantly anxiety and depression. This finding has been confirmed by Salkovskis et al., ${ }^{6}$ who also reported that the high levels of psychological distress persisted at followup a month later.

The mean age of psychiatric A\&E attenders in Singapore (35.5 years) was similar to that in the West (Bristol, 36.0 years; Boston, 31.0 years). Few psychiatric attenders at A\&E were over 65 years of age in the various studies cited. There is also a similarity in clinical features. For example, anxiety and somatic complaints, depressed mood, suicidal attempts or thoughts, and disturbed psychotic behaviour were common presenting symptoms. Approximately $50 \%$ were suffering from nonpsychotic conditions, and $20-25 \%$ were suffering from psychotic disorders.

With regard to the management of patients, $40 \%$ of patients were admitted for further treatment in Singapore. This was similar to the data for British patients, but differed from that for their American 
counterparts, among whom less than a fifth were admitted. Bassuk et al. ${ }^{5}$ attributed the higher admission rates in Bristol compared to Boston to the fact that psychiatric treatment in England was based on the in-patient system.

Patients with psychotic disorders or depression were likely to receive further psychiatric management whether or not they were admitted. However, in the busy setting of the A\&E department, non-psychotic patients with anxiety disorders are likely to be discharged with no further follow-up, unlike those with depression.

As psychiatric problems are not uncommon in the A\&E department, the provision of psychiatric services at the A\&E department of general hospitals in developing countries could form part of a comprehensive mental health care provision for the community. It is hoped that the data presented in this study may assist in the efficient allocation of resources to meet the mental health needs of the rapidly developing nations.

\section{REFERENCES}

1. Lim M.H. (1983) A psychiatric emergency clinic: a study of attendances over six months. British Journal of Psychiatry 143, 460-466.

2. Mindham R.H., Kelleher M.J. \& Birley J.L. (1977) A psychiatric casualty department. Lancet 2, 1067-1068.

3. Bell G., Hindley N., Rajiyah G. \& Rosser R. (1990) Screening for psychiatric morbidity in an accident and emergency department. Archives of Emergency Medicine 7, 155-162.

4. Anstee B.H. (1972) Psychiatry in the casualty department. British Journal of Psychiatry 120, 625629.

5. Bassuk E.L., Winter R. \& Apsler R. (1983) Cross-cultural comparison of British and American psychiatric emergencies. American Journal of Psychiatry 140, 180-184.

6. Salkovskis P.M., Storer D., Atha C. \& Warwick H.M.C. (1990) Psychiatric morbidity in an accident and emergency department-characteristics of patients at presentation and one month follow-up. British Journal of Psychiatry 156, 483-487. 